MATEC Web of Conferences 51, 01013 (2016)

DOI: $10.1051 /$ matecconf/20165101013

C) Owned by the authors, published by EDP Sciences, 2016

\title{
Application and Implementation of the Structured Assembly Process Planning in A Auto Enterprise Based on TS16949
}

\author{
Jiakun Sun ${ }^{1, a}$, Ying Wang ${ }^{1}$, Fupeng Yin $^{1}$ and Huihui Qiu ${ }^{2}$ \\ 1 School of Management Engineering, Shandong Jianzhu University, Jinan, 250101,China \\ 2 Shandong Hoteam Software Co., Ltd. Jinan, 250101, China
}

\begin{abstract}
Process planning is the key link that connects the product design and manufacture. In order to continually improve the effectiveness and efficiency of process planning an auto enterprise in consistent with the process management requirements of TS16949, the requirements for assembly process planning is analysed and the main problems affecting the design and production efficiency are discussed. The infrastructure of the structured process planning and management platform is constructed combing the management technology and TS16949 quality system, then the structured PPPR (product, process, plant and resource)model is reintegrated to analyze the relationships among the objects with the object-oriented method. In the end, with the principles of maintaining conveniently and operating practically, the assembly process planning and management system is implemented according to the characteristics of the process in the enterprise, and the main function modules of the system are described.
\end{abstract}

\section{Introduction}

As one of the most important part in manufacturing enterprises, process planning is the key link that connects the product design and manufacture. As for the automotive industry, it has the characteristics of complex product structure, variety of components, polytrophic process routing and so on[1]. Consequently, the quality and the productivity is closely related to the level of process planning, and the application of CAPP (computer aided process planning)technology[2] is conducive to improve the standardization level and the efficiency of process planning. In recent years, structured process is becoming a tool to organize the product, process, plant, resource (PPPR)[3] and other data resources in a direct and structural manner, which makes it fast, effective and accurate to create and verify the process, and can ensure the standardization and reuse of the process data. It also gets rid of the previous application model where the process planning and management are mainly based on process cards or documents, which limits the support to the process management and be lack of relevance to process data management. Thus, it is becoming increasingly recognized and applied by manufacturing enterprises. The ISO/TS16949 is an ISO technical specification aimed at the development of a quality management system that provides for continual improvement, emphasizing defect prevention and the reduction of variation and waste in the supply chain. A supplier without a valid certificate has little chance of supplying a Tier 1 supplier and certainly no chance of supplying a car manufacturer with

${ }^{\mathrm{a}}$ zpsunjk@sdjzu.edu.cn 
standard parts[4]. Thus, the process planning system should be developed to integrate with TS16949 quality management system[5 6], and, in the application of the system, it must be in accordance with the TS16949 specification, establishing the basic process model of quality management system, combining with the enterprise standard and information technology, to support the standardization and normalization of the processes, so as to improve the quality management efficiency and effectively support the enterprise operation.

\section{Requirement analysis}

The automotive company, located in Wuhu City, Anhui Province, China, was established in 1997. After 18 years of development, it's products have covered the fields of passenger cars, commercial vehicles, etc. For the process planning and management, DELMIA[7] system is applied to support product design and complete the product process planning and verification through three-dimensional model. But there are still deficiencies in the integration of information with the downstream production department and in the combination of the organization and process management, which affect the design and production efficiency, the main problems are as follows:

1) Process planning and management is not standardized and process planning pattern cannot support process planning effectively.

For the process planning pattern, it mainly takes the process card as the core, which emphasis too much on the result of the process planning, so that it can't support the process effectively and only give limited backing to improve the process research, development and management. Meanwhile, the management of process data is chaotic, the data of the product design and process planning is isolated, the process of the process management is not standardized, hence the product design data can't be effectively utilized. Due to the lack of the structured model of management and the poor reuse level, it seriously affect the production schedule.

2) Process planning and management can't meet the fast and personalised market demand

As time goes by, the market requirements becomes fast-changing, and the automotive models are changing, also, the product configuration is becoming more and more complex. Assembly process is still the most time consuming stage among the four major stages of the automotive manufacturing.

3) TS16949 system file can't be managed quickly and effectively

Although the enterprise has passed the TS16949 certification and has a relatively complete management process as well, the TS16949 system management platform applied in the process planning department is not concordant with that of the production department and the functions is limited. As the core tool of TS16949 system , large number of process-related quality documents created by APQP [5] can't be arranged in accordance with the process specification, and the standard specification of the data organization and management can't be met. There are also many faults in the operation process of the TS16949 quality documents for different configurations of the same models, causing a lot of duplication of work.

To sum up, the problems summarized above increase the workload on the staffs, so the automotive enterprise is in urgent need of the support for a structured process planning and management system to improve the level of the standardization and structuralization for process planning and management and increase the efficiency of process planning.

\section{System design}

\subsection{Infrastructure design}

According to the requirements of the enterprise, with the principles of maintaining conveniently and operating practically, the infrastructure for the collaborative process planning and management platform is built combing the management technology and information technology. With the database and file server as the support, it could define different functions through the configuration of the 
object models and data models. The system also provides different interfaces for different users to achieve the process collaboration between the departments of process planning and product design. The system architecture is divided into four layers: basic support layer, maintenance layer, function layer and interface layer. The infrastructure is shown in Figure 1.

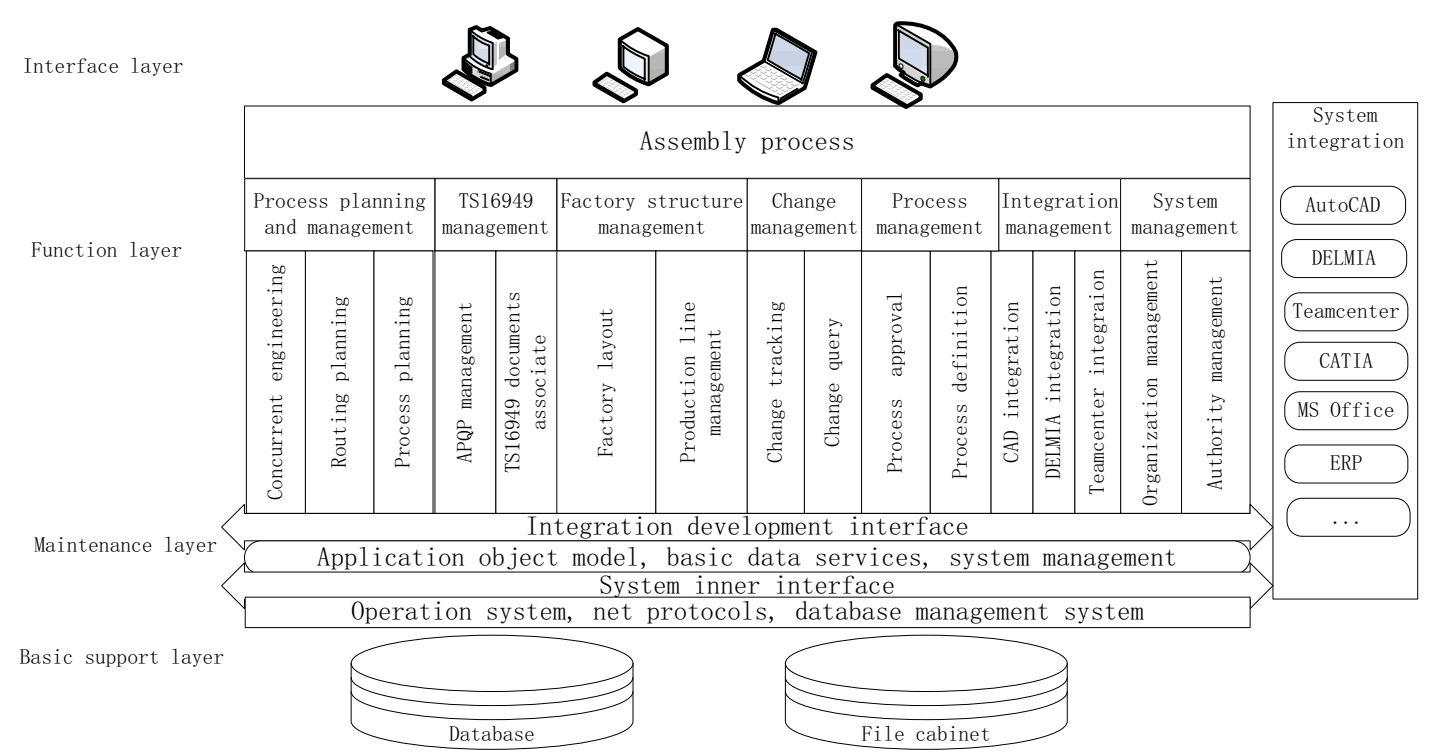

Figure 1. System infrastructure

1) Basic support layer, it is the basis of system development, including network protocols, operating system, a variety of file cabinets and databases. The database mainly used to store the basic data while the file cabinet is mainly used to store some unstructured documents, pictures and videos;

2) Maintenance layer, it is a prerequisite to ensuring the normal application of the system, including the application of the object models, basic data services and system management. Application object model maintenance is the main part of the layer, once the business is to be adjusted, the model will be modified or reconfigured. The system management is mainly to ensure the system security and maintain user's account information.

3) Function layer, it is to form various function modules: assembly process planning and management, TS16949 system management, factory structure management, change management, process management, integration management and system management with the support of the basic support layer and the maintenance layer according to business logic. New function modules can be customized through secondary development or application integration to meet the changing requirements of the enterprise.

4) Interface layer, it makes the interface more user-friendly, providing convenient operation through the application of the principle of man-machine engineering.

Based on the universal process information model, taking the basic system development idea of continual improvement and optimization, it establishes the process planning and management platform with the assembly process planning and management as the core function module that is relatively simple, easy to implement and maintainment.

\subsection{PPPR model of assembly process}

According to the characteristics of the enterprise, the PPPR model is reintegrated to analyze the relationship among the four objects: the product, the process, the plant and the resource, as shown in Figure 2. 
ProductView contains all the attribute information and the structure information of an automotive model in the design phase, which mainly contains two entity objects: component and component structure. The component entity is used to show the attribute information of the components themselves, such as the basic information of name, type, unit, as well as the management information of the CAD diagram, quality requirements, etc. The component structure entity is used to reflect the features of the various models and the structural relations among the various components, which can provide complete information for every department, and it is the basis of PPPR model.

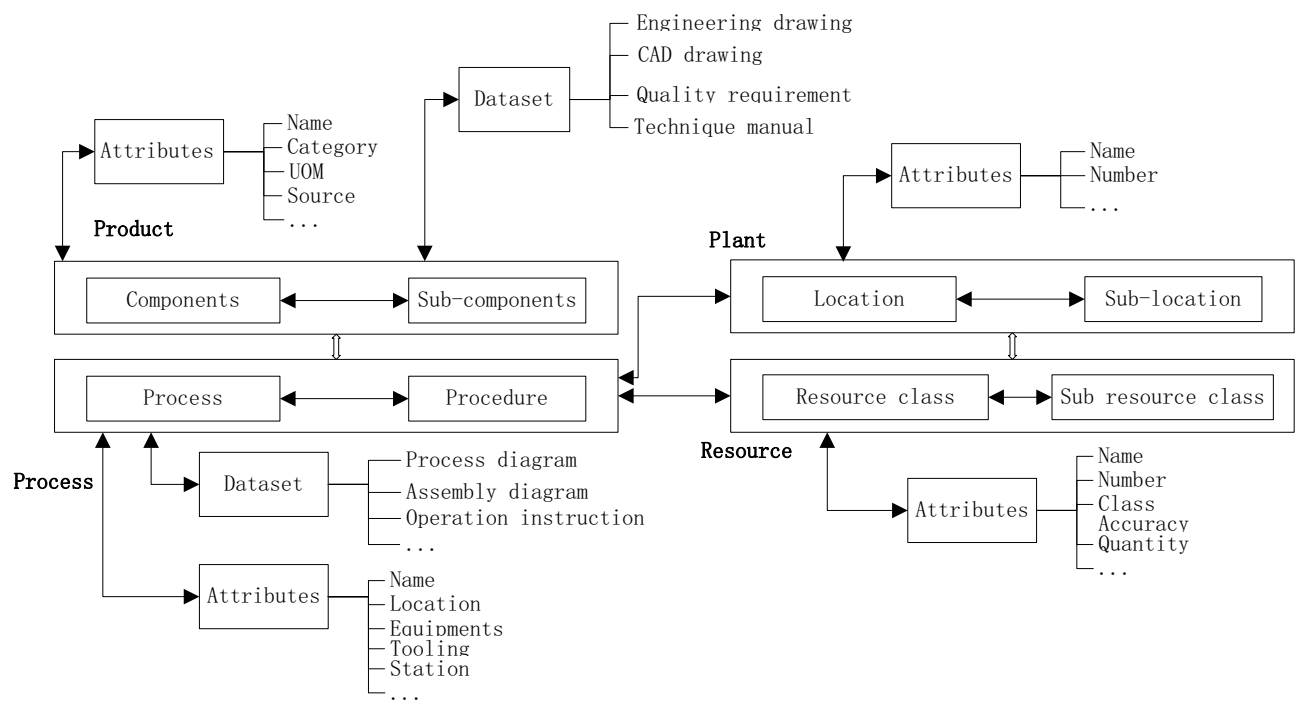

Figure 2. PPPR model

ProcessView is the view from assembly process planning, it contains the content of process planning for various components. The process of the components has independent process attribute information such as name, assembly location, equipment and tooling as well as corresponding technical document information. And, there exists the hierarchical relationships among the processes, the procedures and the operation units, for example, a process is composed of a set of procedures while one procedure consist of one or more operating units.

PlantView contains information about the location of the processed components, and each processing location has its own location attribute information such as location name, number, etc, and there are also the links among different position levels in the location model, such as the relationships among the factories, workshops, production lines and work stations.

ResourceView contains the information of the resources needed for component processing, such as various equipments, tooling, materials, etc, which could be managed through a classification system according to the processing capacity information (such as the processing accuracy, processing size, etc.), the resources' properties (such as name, tool size, etc.) and the relationship among the resources.

ProcessView is the center of the PPPR model, through which the information of man-hours of the components in productview, manufacturing resources information in resources view, the distribution information of the factories workshops, production lines in plantview and the process information in processview can be easily and quickly integrated. It can effectively avoid the information island by making full use of the independent and interrelated relationships among the objects, so as to form a structured process planning and management system which runs through the whole life cycle of a product.

\section{System implementation and application}


Process structure tree is the chain through which the process activities in manufacturing are connected according to the precedence relationship and the subordinate relationship between the process activities. The adjustment of the process structure tree is the foundation of the assembling process planning. Combining with PPPR model, the attributes information, location information, and resources information can be linked together to form the process object. Different hierarchy process objects are corresponding to the process structure tree, all resources are organized according to the purpose, and associated with the process objects, which makes the working process more clear.

1) To establish the assembly process structure tree

On the basis of DELMIA process structure according to the characteristic of enterprise, the process data can be gotten from DELMIA by system integration, then it will be reanalyzed and planned combining with the PPPR model, to establish the assembly process structure tree. The planning principles are as follows: A) the model of factory location should correspond with the location nodes in the process tree in DELMIA; B) location information of different level should correspond with the process objects of different levels. The assembly process structure tree is illustrated in Figure 3.

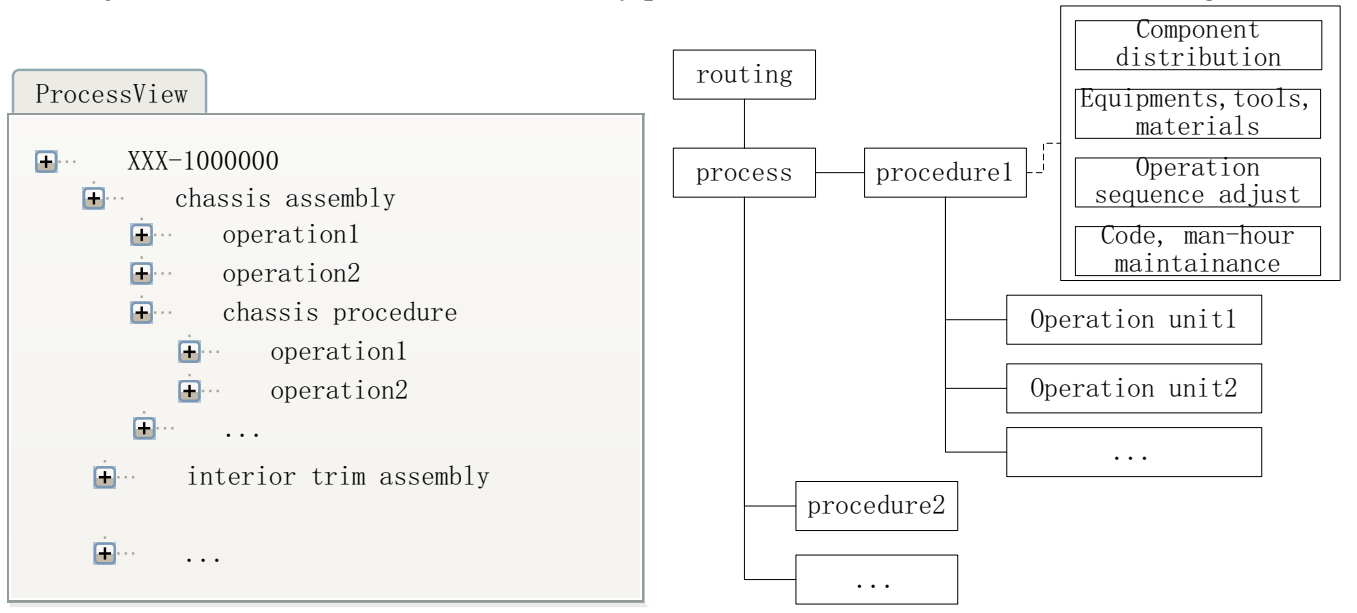

Figure 3. Assembly structure tree

Figure 4. Consummate the process structure tree

2) To consummate the process structure tree

The process structure tree of the first edition needs to be consummated to satisfy the final assembly process planning. By the process object management model, the attribute information of the process objects in process structure tree is adjusted, if design change happens, new process object can be added according to the hierarchy structural relationship. In addition, according to the relationships among all the resources and process objects in PPPR model, the components, production materials and tools are distributed to the process objects of different levels. If the assembly sequence of the operation units under the procedure is changed, according to the relationship between the process object and the process structure tree, the order of the operating units can be adjusted, which can be reflected in the structure tree later. The completed process structure tree can meet the need of the final assembly process planning, as shown in Figure 4.

3) TS16949 documents management

The format of TS16949 documents is standardized, the professional terms and data commonly used in TS16949 quality system are stored in the repository through the application of CAPP knowledge repository, combing with the PPPR model, wherein each type of data resource has its attributes, it may also be classified and managed according to different resource properties. The data sets can be arranged in the form of a tree structure, which is easy to query and extract the relevant system resources. At the same time, the standard templates for each type of system documents, such as process flow diagrams, process FMEA[8] and control plans, are designed, thus, in every stage of APQP [8] implementation, the template of the corresponding class is invoked, the system will automatically pop up the properties box, the content can be selected to fill, then a complete system documents would be created after the execution; In addition, the associated linkage between the 
specific templates can be set, through which one template is filled, the other linked template will be filled with the similar content, when the data is modified or deleted, it also comply with this setup.

\section{Summary}

With assembly process planning and management as the object, the structured assembly process planning and management platform based on TS16949 quality system is developed. According to the requirements from an automotive enterprise assembly process, the main content and process of the assembly process planning and management is analyzed, and the overall structure of the CAPP system is constructed, the assembly process structure tree and process allocation model is designed based on PPPR management model. Also, the main functional modules of the system for process planning and management is analyzed and designed using the object-oriented method. In the end, the CAPP assembly process design and management system is implemented according to the characteristics of the process in the enterprise. Through the establishment and application of common process modules, final assembly process planning can be quickly completed. At the same time, the TS16949 specification is embedded in the structured process planning and applied in the automobile process planning and management. It can not only meet the requirement of the process management from TS16949 quality management system, but also improve the efficiency of the process planning and the use of R \& D resources, reduce the burden of process workers.

\section{Acknowledgements}

This work was financially supported by Electronic and Information Industrial Fund (Contract No. 2012[407]), Ministry of industry and information technology, P.R.China

\section{References}

1. P.Wells, Sustainable business models and the automotive industry: A commentary. IIMB Manage. Rev., 25,12(2013)

2. J.X.Xu, M.Z.Zou, X.G.Kong,etc., Study on the development trend for modern CAPP technology. Manuf. Autom. 9,5(2004)

3. J.Y.Lee, H.S.Kang, G.Y.Kim, S.D.Noh, Concurrent material flow analysis by P3R-driven modeling and simulation in PLM.Comput.Ind. 63,15(2012)

4. C.Michael, ISO/TS 16949 QM in der Automobile industrie umsetzen. Carl Hanser Verlag, (2007)

5. C.P. Kartha. A comparison of ISO 9000:2000 quality system standards, QS9000, ISO/TS 16949 and Baldrige criteria. The TQM Magazine, 16,10 (2004),

6. S.Kwon, K.K.Lee,Y.H.Park. A study on a methodology of integration lean DFSS and advanced product planning(APQP) in ISO/TS16949, Asian J.Qual, 8,9(2013)

7. N.A.M.Salleh, S.Kasolang, A.Jaffar. Simulation of Integrated Total Quality Management (TQM) with lean manufacturing (LM) practices in forming process using Delmia quest. Procedia Eng, 41, 6(2012)

8. A. P. Puvanasvaran, N. Jamibollah, N. Norazlin. Integration of POKA YOKE into process failure mode and effect analysis :a case study. Am.J.Appl.Sci., 11, 11(2014 ) 